\title{
Bounds for the second Hankel determinant of certain univalent functions
}

\author{
See Keong Lee ${ }^{1 *}$, V Ravichandran² and Shamani Supramaniam \\ Dedicated to Professor Hari M Srivastava
}

"Correspondence: sklee@cs.usm.my 'School of Mathematical Sciences, Universiti Sains Malaysia, 11800

USM, Penang, Malaysia

Full list of author information is

available at the end of the article

\begin{abstract}
The estimates for the second Hankel determinant $a_{2} a_{4}-a_{3}^{2}$ of the analytic function $f(z)=z+a_{2} z^{2}+a_{3} z^{3}+\cdots$, for which either $z f^{\prime}(z) / f(z)$ or $1+z f^{\prime \prime}(z) / f^{\prime}(z)$ is subordinate to a certain analytic function, are investigated. The estimates for the Hankel determinant for two other classes are also obtained. In particular, the estimates for the Hankel determinant of strongly starlike, parabolic starlike and lemniscate starlike functions are obtained.

MSC: $30 \mathrm{C} 45 ; 30 \mathrm{C} 80$
\end{abstract}

\section{Introduction}

Let $\mathcal{A}$ denote the class of all analytic functions

$$
f(z)=z+a_{2} z^{2}+a_{3} z^{3}+\cdots
$$

defined on the open unit disk $\mathbb{D}:=\{z \in \mathbb{C}:|z|<1\}$. The Hankel determinants $H_{q}(n)(n=$ $1,2, \ldots, q=1,2, \ldots)$ of the function $f$ are defined by

$$
H_{q}(n):=\left[\begin{array}{cccc}
a_{n} & a_{n+1} & \cdots & a_{n+q-1} \\
a_{n+1} & a_{n+2} & \cdots & a_{n+q} \\
\vdots & \vdots & & \vdots \\
a_{n+q-1} & a_{n+q} & \cdots & a_{n+2 q-2}
\end{array}\right] \quad\left(a_{1}=1\right)
$$

Hankel determinants are useful, for example, in showing that a function of bounded characteristic in $\mathbb{D}$, i.e., a function which is a ratio of two bounded analytic functions with its Laurent series around the origin having integral coefficients, is rational [1]. For the use of Hankel determinants in the study of meromorphic functions, see [2], and various properties of these determinants can be found in [3, Chapter 4]. In 1966, Pommerenke [4] investigated the Hankel determinant of areally mean $p$-valent functions, univalent functions as well as of starlike functions. In [5], he proved that the Hankel determinants of univalent functions satisfy

$$
\left|H_{q}(n)\right|<K n^{-\left(\frac{1}{2}+\beta\right) q+\frac{3}{2}} \quad(n=1,2, \ldots, q=2,3, \ldots),
$$

\section{Springer}

(c) 2013 Lee et al.; licensee Springer. This is an Open Access article distributed under the terms of the Creative Commons Attribution License (http://creativecommons.org/licenses/by/2.0), which permits unrestricted use, distribution, and reproduction in any medium, provided the original work is properly cited. 
where $\beta>1 / 4000$ and $K$ depends only on $q$. Later, Hayman [6] proved that $\left|H_{2}(n)\right|<A n^{1 / 2}$ $(n=1,2, \ldots ; A$ an absolute constant) for areally mean univalent functions. In [7-9], the estimates for the Hankel determinant of areally mean $p$-valent functions were investigated. ElHosh obtained bounds for Hankel determinants of univalent functions with a positive Hayman index $\alpha$ [10] and of $k$-fold symmetric and close-to-convex functions [11]. For bounds on the Hankel determinants of close-to-convex functions, see [12-14]. Noor studied the Hankel determinant of Bazilevic functions in [15] and of functions with bounded boundary rotation in [16-19]. In the recent years, several authors have investigated bounds for the Hankel determinant of functions belonging to various subclasses of univalent and multivalent functions [20-27]. The Hankel determinant $H_{2}(1)=a_{3}-a_{2}^{2}$ is the well-known Fekete-Szegö functional. For results related to this functional, see [28, 29]. The second Hankel determinant $H_{2}(2)$ is given by $H_{2}(2)=a_{2} a_{4}-a_{3}^{2}$.

An analytic function $f$ is subordinate to an analytic function $g$, written $f(z) \prec g(z)$, if there is an analytic function $w: \mathbb{D} \rightarrow \mathbb{D}$ with $w(0)=0$ satisfying $f(z)=g(w(z))$. Ma and Minda [30] unified various subclasses of starlike $\left(\mathcal{S}^{*}\right)$ and convex functions $(\mathcal{C})$ by requiring that either of the quantity $z f^{\prime}(z) / f(z)$ or $1+z f^{\prime \prime}(z) / f^{\prime}(z)$ is subordinate to a function $\varphi$ with a positive real part in the unit disk $\mathbb{D}, \varphi(0)=1, \varphi^{\prime}(0)>0, \varphi$ maps $\mathbb{D}$ onto a region starlike with respect to 1 and symmetric with respect to the real axis. He obtained distortion, growth and covering estimates as well as bounds for the initial coefficients of the unified classes.

The bounds for the second Hankel determinant $H_{2}(2)=a_{2} a_{4}-a_{3}^{2}$ are obtained for functions belonging to these subclasses of Ma-Minda starlike and convex functions in Section 2. In Section 3, the problem is investigated for two other related classes defined by subordination. In proving our results, we do not assume the univalence or starlikeness of $\varphi$ as they were required only in obtaining the distortion, growth estimates and the convolution theorems. The classes introduced by subordination naturally include several wellknown classes of univalent functions and the results for some of these special classes are indicated as corollaries.

Let $\mathcal{P}$ be the class of functions with positive real part consisting of all analytic functions $p: \mathbb{D} \rightarrow \mathbb{C}$ satisfying $p(0)=1$ and $\operatorname{Re} p(z)>0$. We need the following results about the functions belonging to the class $\mathcal{P}$.

Lemma 1 [31] If the function $p \in \mathcal{P}$ is given by the series

$$
p(z)=1+c_{1} z+c_{2} z^{2}+c_{3} z^{3}+\cdots,
$$

then the following sharp estimate holds:

$$
\left|c_{n}\right| \leq 2 \quad(n=1,2, \ldots)
$$

Lemma 2 [32] If the function $p \in \mathcal{P}$ is given by the series (2), then

$$
\begin{aligned}
& 2 c_{2}=c_{1}^{2}+x\left(4-c_{1}^{2}\right), \\
& 4 c_{3}=c_{1}^{3}+2\left(4-c_{1}^{2}\right) c_{1} x-c_{1}\left(4-c_{1}^{2}\right) x^{2}+2\left(4-c_{1}^{2}\right)\left(1-|x|^{2}\right) z,
\end{aligned}
$$

for some $x, z$ with $|x| \leq 1$ and $|z| \leq 1$. 


\section{Second Hankel determinant of Ma-Minda starlike/convex functions}

Subclasses of starlike functions are characterized by the quantity $z f^{\prime}(z) / f(z)$ lying in some domain in the right half-plane. For example, $f$ is strongly starlike of order $\beta$ if $z f^{\prime}(z) / f(z)$ lies in a sector $|\arg w|<\beta \pi / 2$, while it is starlike of order $\alpha$ if $z f^{\prime}(z) / f(z)$ lies in the halfplane $\operatorname{Re} w>\alpha$. The various subclasses of starlike functions were unified by subordination in [30]. The following definition of the class of Ma-Minda starlike functions is the same as the one in [30] except for the omission of starlikeness assumption of $\varphi$.

Definition 1 Let $\varphi: \mathbb{D} \rightarrow \mathbb{C}$ be analytic, and let the Maclaurin series of $\varphi$ be given by

$$
\varphi(z)=1+B_{1} z+B_{2} z^{2}+B_{3} z^{3}+\cdots \quad\left(B_{1}, B_{2} \in \mathbb{R}, B_{1}>0\right) .
$$

The class $\mathcal{S}^{*}(\varphi)$ of Ma-Minda starlike functions with respect to $\varphi$ consists of functions $f \in \mathcal{A}$ satisfying the subordination

$$
\frac{z f^{\prime}(z)}{f(z)} \prec \varphi(z)
$$

For the function $\varphi$ given by $\varphi_{\alpha}(z):=(1+(1-2 \alpha) z) /(1-z), 0<\alpha \leq 1$, the class $\mathcal{S}^{*}(\alpha):=$ $\mathcal{S}^{*}\left(\varphi_{\alpha}\right)$ is the well-known class of starlike functions of order $\alpha$. Let

$$
\varphi_{P A R}(z):=1+\frac{2}{\pi^{2}}\left(\log \frac{1+\sqrt{z}}{1-\sqrt{z}}\right)^{2} .
$$

Then the class

$$
\mathcal{S}_{P}^{*}:=\mathcal{S}^{*}\left(\varphi_{\text {PAR }}\right)=\left\{f \in \mathcal{A}: \operatorname{Re}\left(\frac{z f^{\prime}(z)}{f(z)}\right)>\left|\frac{z f^{\prime}(z)}{f(z)}-1\right|\right\}
$$

is the parabolic starlike functions introduced by Rønning [33]. For a survey of parabolic starlike functions and the related class of uniformly convex functions, see [34]. For $0<\beta \leq 1$, the class

$$
\mathcal{S}_{\beta}^{*}:=\mathcal{S}^{*}\left(\left(\frac{1+z}{1-z}\right)^{\beta}\right)=\left\{f \in \mathcal{A}:\left|\arg \left(\frac{z f^{\prime}(z)}{f(z)}\right)\right|<\frac{\beta \pi}{2}\right\}
$$

is the familiar class of strongly starlike functions of order $\beta$. The class

$$
\mathcal{S}_{L}^{*}:=\mathcal{S}^{*}(\sqrt{1+z})=\left\{f \in \mathcal{A}:\left|\left(\frac{z f^{\prime}(z)}{f(z)}\right)^{2}-1\right|<1\right\}
$$

is the class of lemniscate starlike functions studied in [35].

Theorem 1 Let the function $f \in \mathcal{S}^{*}(\varphi)$ be given by (1).

1. If $B_{1}, B_{2}$ and $B_{3}$ satisfy the conditions

$$
\left|B_{2}\right| \leq B_{1}, \quad\left|4 B_{1} B_{3}-B_{1}^{4}-3 B_{2}^{2}\right|-3 B_{1}^{2} \leq 0,
$$


then the second Hankel determinant satisfies

$$
\left|a_{2} a_{4}-a_{3}^{2}\right| \leq \frac{B_{1}^{2}}{4}
$$

2. If $B_{1}, B_{2}$ and $B_{3}$ satisfy the conditions

$$
\left|B_{2}\right| \geq B_{1}, \quad\left|4 B_{1} B_{3}-B_{1}^{4}-3 B_{2}^{2}\right|-B_{1}\left|B_{2}\right|-2 B_{1}^{2} \geq 0,
$$

or the conditions

$$
\left|B_{2}\right| \leq B_{1}, \quad\left|4 B_{1} B_{3}-B_{1}^{4}-3 B_{2}^{2}\right|-3 B_{1}^{2} \geq 0,
$$

then the second Hankel determinant satisfies

$$
\left|a_{2} a_{4}-a_{3}^{2}\right| \leq \frac{1}{12}\left|4 B_{1} B_{3}-B_{1}^{4}-3 B_{2}^{2}\right| .
$$

3. If $B_{1}, B_{2}$ and $B_{3}$ satisfy the conditions

$$
\left|B_{2}\right|>B_{1}, \quad\left|4 B_{1} B_{3}-B_{1}^{4}-3 B_{2}^{2}\right|-B_{1}\left|B_{2}\right|-2 B_{1}^{2} \leq 0,
$$

then the second Hankel determinant satisfies

$$
\left|a_{2} a_{4}-a_{3}^{2}\right| \leq \frac{B_{1}^{2}}{12}\left(\frac{3\left|4 B_{1} B_{3}-B_{1}^{4}-3 B_{2}^{2}\right|-4 B_{1}\left|B_{2}\right|+4 B_{1}^{2}-B_{2}^{2}}{\left|4 B_{1} B_{3}-B_{1}^{4}-3 B_{2}^{2}\right|-2 B_{1}\left|B_{2}\right|-B_{1}^{2}}\right)
$$

Proof Since $f \in \mathcal{S}^{*}(\varphi)$, there exists an analytic function $w$ with $w(0)=0$ and $|w(z)|<1$ in $\mathbb{D}$ such that

$$
\frac{z f^{\prime}(z)}{f(z)}=\varphi(w(z)) .
$$

Define the functions $p_{1}$ by

$$
p_{1}(z):=\frac{1+w(z)}{1-w(z)}=1+c_{1} z+c_{2} z^{2}+\cdots
$$

or, equivalently,

$$
w(z)=\frac{p_{1}(z)-1}{p_{1}(z)+1}=\frac{1}{2}\left(c_{1} z+\left(c_{2}-\frac{c_{1}^{2}}{2}\right) z^{2}+\cdots\right) .
$$

Then $p_{1}$ is analytic in $\mathbb{D}$ with $p_{1}(0)=1$ and has a positive real part in $\mathbb{D}$. By using $(8)$ together with (6), it is evident that

$$
\varphi\left(\frac{p_{1}(z)-1}{p_{1}(z)+1}\right)=1+\frac{1}{2} B_{1} c_{1} z+\left(\frac{1}{2} B_{1}\left(c_{2}-\frac{c_{1}^{2}}{2}\right)+\frac{1}{4} B_{2} c_{1}^{2}\right) z^{2}+\cdots .
$$

Since

$$
\frac{z f^{\prime}(z)}{f(z)}=1+a_{2} z+\left(-a_{2}^{2}+2 a_{3}\right) z^{2}+\left(3 a_{4}-3 a_{2} a_{3}+a_{2}^{3}\right) z^{3}+\cdots,
$$


it follows by (7), (9) and (10) that

$$
\begin{aligned}
a_{2}= & \frac{B_{1} c_{1}}{2}, \\
a_{3}= & \frac{1}{8}\left[\left(B_{1}^{2}-B_{1}+B_{2}\right) c_{1}^{2}+2 B_{1} c_{2}\right], \\
a_{4}= & \frac{1}{48}\left[\left(-4 B_{2}+2 B_{1}+B_{1}^{3}-3 B_{1}^{2}+3 B_{1} B_{2}+2 B_{3}\right) c_{1}^{3}\right. \\
& \left.+2\left(3 B_{1}^{2}-4 B_{1}+4 B_{2}\right) c_{1} c_{2}+8 B_{1} c_{3}\right] .
\end{aligned}
$$

Therefore

$$
a_{2} a_{4}-a_{3}^{2}=\frac{B_{1}}{96}\left[c_{1}^{4}\left(-\frac{B_{1}^{3}}{2}+\frac{B_{1}}{2}-B_{2}+2 B_{3}-\frac{3 B_{2}^{2}}{2 B_{1}}\right)+2 c_{2} c_{1}^{2}\left(B_{2}-B_{1}\right)+8 B_{1} c_{1} c_{3}-6 B_{1} c_{2}^{2}\right] .
$$

Let

$$
\begin{aligned}
& d_{1}=8 B_{1}, \quad d_{2}=2\left(B_{2}-B_{1}\right), \\
& d_{3}=-6 B_{1}, \quad d_{4}=-\frac{B_{1}^{3}}{2}+\frac{B_{1}}{2}-B_{2}+2 B_{3}-\frac{3 B_{2}^{2}}{2 B_{1}}, \\
& T=\frac{B_{1}}{96} .
\end{aligned}
$$

Then

$$
\left|a_{2} a_{4}-a_{3}^{2}\right|=T\left|d_{1} c_{1} c_{3}+d_{2} c_{1}^{2} c_{2}+d_{3} c_{2}^{2}+d_{4} c_{1}^{4}\right|
$$

Since the function $p\left(e^{i \theta} z\right)(\theta \in \mathbb{R})$ is in the class $\mathcal{P}$ for any $p \in \mathcal{P}$, there is no loss of generality in assuming $c_{1}>0$. Write $c_{1}=c, c \in[0,2]$. Substituting the values of $c_{2}$ and $c_{3}$ respectively from (4) and (5) in (12), we obtain

$$
\begin{aligned}
\left|a_{2} a_{4}-a_{3}^{2}\right|= & \frac{T}{4} \mid c^{4}\left(d_{1}+2 d_{2}+d_{3}+4 d_{4}\right)+2 x c^{2}\left(4-c^{2}\right)\left(d_{1}+d_{2}+d_{3}\right) \\
& +\left(4-c^{2}\right) x^{2}\left(-d_{1} c^{2}+d_{3}\left(4-c^{2}\right)\right)+2 d_{1} c\left(4-c^{2}\right)\left(1-|x|^{2}\right) z \mid .
\end{aligned}
$$

Replacing $|x|$ by $\mu$ and substituting the values of $d_{1}, d_{2}, d_{3}$ and $d_{4}$ from (11) yield

$$
\begin{aligned}
\left|a_{2} a_{4}-a_{3}^{2}\right| \leq & \frac{T}{4}\left[c^{4}\left|-2 B_{1}^{3}+8 B_{3}-6 \frac{B_{2}^{2}}{B_{1}}\right|+4\left|B_{2}\right| \mu c^{2}\left(4-c^{2}\right)\right. \\
& \left.+\mu^{2}\left(4-c^{2}\right)\left(2 B_{1} c^{2}+24 B_{1}\right)+16 B_{1} c\left(4-c^{2}\right)\left(1-\mu^{2}\right)\right] \\
= & T\left[\frac{c^{4}}{4}\left|-2 B_{1}^{3}+8 B_{3}-6 \frac{B_{2}^{2}}{B_{1}}\right|+4 B_{1} c\left(4-c^{2}\right)+\left|B_{2}\right|\left(4-c^{2}\right) \mu c^{2}\right. \\
& \left.+\frac{B_{1}}{2} \mu^{2}\left(4-c^{2}\right)(c-6)(c-2)\right] \\
\equiv & F(c, \mu) .
\end{aligned}
$$


Note that for $(c, \mu) \in[0,2] \times[0,1]$, differentiating $F(c, \mu)$ in (13) partially with respect to $\mu$ yields

$$
\frac{\partial F}{\partial \mu}=T\left[\left|B_{2}\right|\left(4-c^{2}\right)+B_{1} \mu\left(4-c^{2}\right)(c-2)(c-6)\right] .
$$

Then, for $0<\mu<1$ and for any fixed $c$ with $0<c<2$, it is clear from (14) that $\frac{\partial F}{\partial \mu}>0$, that is, $F(c, \mu)$ is an increasing function of $\mu$. Hence, for fixed $c \in[0,2]$, the maximum of $F(c, \mu)$ occurs at $\mu=1$, and

$$
\max F(c, \mu)=F(c, 1) \equiv G(c) .
$$

Also note that

$$
G(c)=\frac{B_{1}}{96}\left[\frac{c^{4}}{4}\left(\left|-2 B_{1}^{3}+8 B_{3}-6 \frac{B_{2}^{2}}{B_{1}}\right|-4\left|B_{2}\right|-2 B_{1}\right)+4 c^{2}\left(\left|B_{2}\right|-B_{1}\right)+24 B_{1}\right] .
$$

Let

$$
\begin{aligned}
& P=\frac{1}{4}\left(\left|-2 B_{1}^{3}+8 B_{3}-6 \frac{B_{2}^{2}}{B_{1}}\right|-4\left|B_{2}\right|-2 B_{1}\right), \\
& Q=4\left(\left|B_{2}\right|-B_{1}\right), \\
& R=24 B_{1} .
\end{aligned}
$$

Since

$$
\max _{0 \leq t \leq 4}\left(P t^{2}+Q t+R\right)= \begin{cases}R, & Q \leq 0, P \leq-\frac{Q}{4} ; \\ 16 P+4 Q+R, & Q \geq 0, P \geq-\frac{Q}{8} \text { or } Q \leq 0, P \geq-\frac{Q}{4} ; \\ \frac{4 P R-Q^{2}}{4 P}, & Q>0, P \leq-\frac{Q}{8},\end{cases}
$$

we have

$$
\left|a_{2} a_{4}-a_{3}^{2}\right| \leq \frac{B_{1}}{96} \begin{cases}R, & Q \leq 0, P \leq-\frac{Q}{4} \\ 16 P+4 Q+R, & Q \geq 0, P \geq-\frac{Q}{8} \text { or } Q \leq 0, P \geq-\frac{Q}{4} \\ \frac{4 P R-Q^{2}}{4 P}, & Q>0, P \leq-\frac{Q}{8}\end{cases}
$$

where $P, Q, R$ are given by (15).

Remark 1 When $B_{1}=B_{2}=B_{3}=2$, Theorem 1 reduces to [24, Theorem 3.1].

\section{Corollary 1}

1. If $\in \mathcal{S}^{*}(\alpha)$, then $\left|a_{2} a_{4}-a_{3}^{2}\right| \leq(1-\alpha)^{2}$.

2. If $\in \mathcal{S}_{L}^{*}$, then $\left|a_{2} a_{4}-a_{3}^{2}\right| \leq 1 / 16=0.0625$.

3. If $\in \mathcal{S}_{P}^{*}$, then $\left|a_{2} a_{4}-a_{3}^{2}\right| \leq 16 / \pi^{4} \approx 0.164255$.

4. If $\in \mathcal{S}_{\beta}^{*}$, then $\left|a_{2} a_{4}-a_{3}^{2}\right| \leq \beta^{2}$. 
Definition 2 Let $\varphi: \mathbb{D} \rightarrow \mathbb{C}$ be analytic, and let $\varphi(z)$ be given as in (6). The class $\mathcal{C}(\varphi)$ of Ma-Minda convex functions with respect to $\varphi$ consists of functions $f$ satisfying the subordination

$$
1+\frac{z f^{\prime \prime}(z)}{f^{\prime}(z)} \prec \varphi(z)
$$

Theorem 2 Let the function $f \in \mathcal{C}(\varphi)$ be given by (1).

1. If $B_{1}, B_{2}$ and $B_{3}$ satisfy the conditions

$$
B_{1}^{2}+4\left|B_{2}\right|-2 B_{1} \leq 0, \quad\left|6 B_{1} B_{3}+B_{1}^{2} B_{2}-B_{1}^{4}-4 B_{2}^{2}\right|-4 B_{1}^{2} \leq 0,
$$

then the second Hankel determinant satisfies

$$
\left|a_{2} a_{4}-a_{3}^{2}\right| \leq \frac{B_{1}^{2}}{36}
$$

2. If $B_{1}, B_{2}$ and $B_{3}$ satisfy the conditions

$$
B_{1}^{2}+4\left|B_{2}\right|-2 B_{1} \geq 0, \quad 2\left|6 B_{1} B_{3}+B_{1}^{2} B_{2}-B_{1}^{4}-4 B_{2}^{2}\right|-B_{1}^{3}-4 B_{1}\left|B_{2}\right|-6 B_{1}^{2} \geq 0,
$$

or the conditions

$$
B_{1}^{2}+4\left|B_{2}\right|-2 B_{1} \leq 0, \quad\left|6 B_{1} B_{3}+B_{1}^{2} B_{2}-B_{1}^{4}-4 B_{2}^{2}\right|-4 B_{1}^{2} \geq 0,
$$

then the second Hankel determinant satisfies

$$
\left|a_{2} a_{4}-a_{3}^{2}\right| \leq \frac{1}{144}\left|6 B_{1} B_{3}+B_{1}^{2} B_{2}-B_{1}^{4}-4 B_{2}^{2}\right|
$$

3. If $B_{1}, B_{2}$ and $B_{3}$ satisfy the conditions

$$
B_{1}^{2}+4\left|B_{2}\right|-2 B_{1}>0, \quad 2\left|6 B_{1} B_{3}+B_{1}^{2} B_{2}-B_{1}^{4}-4 B_{2}^{2}\right|-B_{1}^{3}-4 B_{1}\left|B_{2}\right|-6 B_{1}^{2} \leq 0,
$$

then the second Hankel determinant satisfies

$$
\left|a_{2} a_{4}-a_{3}^{2}\right| \leq \frac{B_{1}^{2}}{576}\left(\begin{array}{c}
16\left|6 B_{1} B_{3}+B_{1}^{2} B_{2}-B_{1}^{4}-4 B_{2}^{2}\right|-12 B_{1}^{3}-48 B_{1}\left|B_{2}\right| \\
\frac{-36 B_{1}^{2}-B_{1}^{4}-8 B_{1}^{2}\left|B_{2}\right|-16 B_{2}^{2}}{\left|6 B_{1} B_{3}+B_{1}^{2} B_{2}-B_{1}^{4}-4 B_{2}^{2}\right|-B_{1}^{3}-4 B_{1}\left|B_{2}\right|-2 B_{1}^{2}}
\end{array}\right)
$$

Proof Since $f \in \mathcal{C}(\varphi)$, there exists an analytic function $w$ with $w(0)=0$ and $|w(z)|<1$ in $\mathbb{D}$ such that

$$
1+\frac{z f^{\prime \prime}(z)}{f^{\prime}(z)}=\varphi(w(z))
$$

Since

$$
1+\frac{z f^{\prime \prime}(z)}{f^{\prime}(z)}=1+2 a_{2} z+\left(-4 a_{2}^{2}+6 a_{3}\right) z^{2}+\left(8 a_{2}^{3}-18 a_{2} a_{3}+12 a_{4}\right) z^{3}+\cdots,
$$


equations (9), (17) and (18) yield

$$
\begin{aligned}
a_{2}= & \frac{B_{1} c_{1}}{4} \\
a_{3}= & \frac{1}{24}\left[\left(B_{1}^{2}-B_{1}+B_{2}\right) c_{1}^{2}+2 B_{1} c_{2}\right], \\
a_{4}= & \frac{1}{192}\left[\left(-4 B_{2}+2 B_{1}+B_{1}^{3}-3 B_{1}^{2}+3 B_{1} B_{2}+2 B_{3}\right) c_{1}^{3}\right. \\
& \left.+2\left(3 B_{1}^{2}-4 B_{1}+4 B_{2}\right) c_{1} c_{2}+8 B_{1} c_{3}\right] .
\end{aligned}
$$

Therefore

$$
\begin{aligned}
a_{2} a_{4}-a_{3}^{2}= & \frac{B_{1}}{768}\left[c_{1}^{4}\left(-\frac{4}{3} B_{2}+\frac{2}{3} B_{1}-\frac{1}{3} B_{1}^{3}-\frac{1}{3} B_{1}^{2}+\frac{1}{3} B_{1} B_{2}+2 B_{3}-\frac{4}{3} \frac{B_{2}^{2}}{B_{1}}\right)\right. \\
& \left.+\frac{2}{3} c_{2} c_{1}^{2}\left(B_{1}^{2}-4 B_{1}+4 B_{2}\right)+8 B_{1} c_{1} c_{3}-\frac{16}{3} B_{1} c_{2}^{2}\right] .
\end{aligned}
$$

By writing

$$
\begin{aligned}
& d_{1}=8 B_{1}, \quad d_{2}=\frac{2}{3}\left(B_{1}^{2}-4 B_{1}+4 B_{2}\right), \\
& d_{3}=-\frac{16}{3} B_{1}, \quad d_{4}=-\frac{4}{3} B_{2}+\frac{2}{3} B_{1}-\frac{1}{3} B_{1}^{3}-\frac{1}{3} B_{1}^{2}+\frac{1}{3} B_{1} B_{2}+2 B_{3}-\frac{4}{3} \frac{B_{2}^{2}}{B_{1}}, \\
& T=\frac{B_{1}}{768},
\end{aligned}
$$

we have

$$
\left|a_{2} a_{4}-a_{3}^{2}\right|=T\left|d_{1} c_{1} c_{3}+d_{2} c_{1}^{2} c_{2}+d_{3} c_{2}^{2}+d_{4} c_{1}^{4}\right|
$$

Similar as in Theorems 1, it follows from (4) and (5) that

$$
\begin{aligned}
\left|a_{2} a_{4}-a_{3}^{2}\right|= & \frac{T}{4} \mid c^{4}\left(d_{1}+2 d_{2}+d_{3}+4 d_{4}\right)+2 x c^{2}\left(4-c^{2}\right)\left(d_{1}+d_{2}+d_{3}\right) \\
& +\left(4-c^{2}\right) x^{2}\left(-d_{1} c^{2}+d_{3}\left(4-c^{2}\right)\right)+2 d_{1} c\left(4-c^{2}\right)\left(1-|x|^{2}\right) z \mid .
\end{aligned}
$$

Replacing $|x|$ by $\mu$ and then substituting the values of $d_{1}, d_{2}, d_{3}$ and $d_{4}$ from (19) yield

$$
\begin{aligned}
\left|a_{2} a_{4}-a_{3}^{2}\right| \leq & \frac{T}{4}\left[c^{4}\left|-\frac{4}{3} B_{1}^{3}+\frac{4}{3} B_{1} B_{2}+8 B_{3}-\frac{16}{3} \frac{B_{2}^{2}}{B_{1}}\right|\right. \\
& +2 \mu c^{2}\left(4-c^{2}\right)\left(\frac{2}{3} B_{1}^{2}+\frac{8}{3}\left|B_{2}\right|\right) \\
& \left.+\mu^{2}\left(4-c^{2}\right)\left(\frac{8}{3} B_{1} c^{2}+\frac{64}{3} B_{1}\right)+16 B_{1} c\left(4-c^{2}\right)\left(1-\mu^{2}\right)\right] \\
= & T\left[\frac{c^{4}}{3}\left|-B_{1}^{3}+B_{1} B_{2}+6 B_{3}-4 \frac{B_{2}^{2}}{B_{1}}\right|+4 B_{1} c\left(4-c^{2}\right)\right. \\
& +\frac{1}{3} \mu c^{2}\left(4-c^{2}\right)\left(B_{1}^{2}+4\left|B_{2}\right|\right)
\end{aligned}
$$




$$
\begin{aligned}
& \left.+\frac{2 B_{1}}{3} \mu^{2}\left(4-c^{2}\right)(c-4)(c-2)\right] \\
\equiv & F(c, \mu) .
\end{aligned}
$$

Again, differentiating $F(c, \mu)$ in (21) partially with respect to $\mu$ yields

$$
\frac{\partial F}{\partial \mu}=T\left[\frac{c^{2}}{3}\left(4-c^{2}\right)\left(B_{1}^{2}+4\left|B_{2}\right|\right)+\frac{4 B_{1}}{3} \mu\left(4-c^{2}\right)(c-4)(c-2)\right] .
$$

It is clear from (22) that $\frac{\partial F}{\partial \mu}>0$. Thus $F(c, \mu)$ is an increasing function of $\mu$ for $0<\mu<1$ and for any fixed $c$ with $0<c<2$. So, the maximum of $F(c, \mu)$ occurs at $\mu=1$ and

$$
\max F(c, \mu)=F(c, 1) \equiv G(c) .
$$

Note that

$$
\begin{aligned}
G(c)= & T\left[\frac{c^{4}}{3}\left(\left|-B_{1}^{3}+B_{1} B_{2}+6 B_{3}-4 \frac{B_{2}^{2}}{B_{1}}\right|-B_{1}^{2}-4\left|B_{2}\right|-2 B_{1}\right)\right. \\
& \left.+\frac{4}{3} c^{2}\left(B_{1}^{2}+4\left|B_{2}\right|-2 B_{1}\right)+\frac{64}{3} B_{1}\right] .
\end{aligned}
$$

Let

$$
\begin{aligned}
& P=\frac{1}{3}\left(\left|-B_{1}^{3}+B_{1} B_{2}+6 B_{3}-4 \frac{B_{2}^{2}}{B_{1}}\right|-B_{1}^{2}-4\left|B_{2}\right|-2 B_{1}\right), \\
& Q=\frac{4}{3}\left(B_{1}^{2}+4\left|B_{2}\right|-2 B_{1}\right), \\
& R=\frac{64}{3} B_{1} .
\end{aligned}
$$

By using (16), we have

$$
\left|a_{2} a_{4}-a_{3}^{2}\right| \leq \frac{B_{1}}{768} \begin{cases}R, & Q \leq 0, P \leq-\frac{Q}{4} \\ 16 P+4 Q+R, & Q \geq 0, P \geq-\frac{Q}{8} \text { or } Q \leq 0, P \geq-\frac{Q}{4} \\ \frac{4 P R-Q^{2}}{4 P}, & Q>0, P \leq-\frac{Q}{8}\end{cases}
$$

where $P, Q, R$ are given in (23).

Remark 2 For the choice of $\varphi(z)=(1+z) /(1-z)$, Theorem 2 reduces to [24, Theorem 3.2].

\section{Further results on the second Hankel determinant}

Definition 3 Let $\varphi: \mathbb{D} \rightarrow \mathbb{C}$ be analytic, and let $\varphi(z)$ be as given in (6). Let $0 \leq \gamma \leq 1$ and $\tau \in \mathbb{C} \backslash\{0\}$. A function $f \in \mathcal{A}$ is in the class $\mathcal{R}_{\gamma}^{\tau}(\varphi)$ if it satisfies the following subordination:

$$
1+\frac{1}{\tau}\left(f^{\prime}(z)+\gamma z f^{\prime \prime}(z)-1\right) \prec \varphi(z)
$$


Theorem 3 Let $0 \leq \gamma \leq 1, \tau \in \mathbb{C} \backslash\{0\}$, and let the function $f$ as in (1) be in the class $\mathcal{R}_{\gamma}^{\tau}(\varphi)$. Also, let

$$
p=\frac{8}{9} \frac{(1+\gamma)(1+3 \gamma)}{(1+2 \gamma)^{2}} .
$$

1. If $B_{1}, B_{2}$ and $B_{3}$ satisfy the conditions

$$
2\left|B_{2}\right|(1-p)+B_{1}(1-2 p) \leq 0, \quad\left|B_{1} B_{3}-p B_{2}^{2}\right|-p B_{1}^{2} \leq 0,
$$

then the second Hankel determinant satisfies

$$
\left|a_{2} a_{4}-a_{3}^{2}\right| \leq \frac{|\tau|^{2} B_{1}^{2}}{9(1+2 \gamma)^{2}}
$$

2. If $B_{1}, B_{2}$ and $B_{3}$ satisfy the conditions

$$
2\left|B_{2}\right|(1-p)+B_{1}(1-2 p) \geq 0, \quad 2\left|B_{1} B_{3}-p B_{2}^{2}\right|-2(1-p) B_{1}\left|B_{2}\right|-B_{1} \geq 0,
$$

or the conditions

$$
2\left|B_{2}\right|(1-p)+B_{1}(1-2 p) \leq 0, \quad\left|B_{1} B_{3}-p B_{2}^{2}\right|-B_{1}^{2} \geq 0
$$

then the second Hankel determinant satisfies

$$
\left|a_{2} a_{4}-a_{3}^{2}\right| \leq \frac{|\tau|^{2}}{8(1+\gamma)(1+3 \gamma)}\left|B_{3} B_{1}-p B_{2}^{2}\right|
$$

3. If $B_{1}, B_{2}$ and $B_{3}$ satisfy the conditions

$$
2\left|B_{2}\right|(1-p)+B_{1}(1-2 p)>0, \quad 2\left|B_{1} B_{3}-p B_{2}^{2}\right|-2(1-p) B_{1}\left|B_{2}\right|-B_{1}^{2} \leq 0,
$$

then the second Hankel determinant satisfies

$$
\begin{aligned}
\left|a_{2} a_{4}-a_{3}^{2}\right| \leq & \frac{|\tau|^{2} B_{1}^{2}}{32(1+\gamma)(1+3 \gamma)} \\
& \times\left(\begin{array}{c}
4 p\left|B_{3} B_{1}-p B_{2}^{2}\right|-4(1-p) B_{1}\left[\left|B_{2}\right|(3-2 p)+B_{1}\right] \\
\frac{-4 B_{2}^{2}(1-p)^{2}-B_{1}^{2}(1-2 p)^{2}}{\left|B_{3} B_{1}-p B_{2}^{2}\right|-(1-p) B_{1}\left(2\left|B_{2}\right|+B_{1}\right)}
\end{array}\right) .
\end{aligned}
$$

Proof For $f \in \mathcal{R}_{\gamma}^{\tau}(\varphi)$, there exists an analytic function $w$ with $w(0)=0$ and $|w(z)|<1$ in $\mathbb{D}$ such that

$$
1+\frac{1}{\tau}\left(f^{\prime}(z)+\gamma z f^{\prime \prime}(z)-1\right)=\varphi(w(z)) .
$$


Since $f$ has the Maclaurin series given by (1), a computation shows that

$$
\begin{aligned}
1+ & \frac{1}{\tau}\left(f^{\prime}(z)+\gamma z f^{\prime \prime}(z)-1\right) \\
& =1+\frac{2 a_{2}(1+\gamma)}{\tau} z+\frac{3 a_{3}(1+2 \gamma)}{\tau} z^{2}+\frac{4 a_{4}(1+3 \gamma)}{\tau} z^{3}+\cdots .
\end{aligned}
$$

It follows from (24), (9) and (25) that

$$
\begin{aligned}
& a_{2}=\frac{\tau B_{1} c_{1}}{4(1+\gamma)} \\
& a_{3}=\frac{\tau B_{1}}{12(1+2 \gamma)}\left[2 c_{2}+c_{1}^{2}\left(\frac{B_{2}}{B_{1}}-1\right)\right], \\
& a_{4}=\frac{\tau}{32(1+3 \gamma)}\left[B_{1}\left(4 c_{3}-4 c_{1} c_{2}+c_{1}^{3}\right)+2 B_{2} c_{1}\left(2 c_{2}-c_{1}^{2}\right)+B_{3} c_{1}^{3}\right] .
\end{aligned}
$$

Therefore

$$
\begin{aligned}
a_{2} a_{4} & -a_{3}^{2} \\
= & \frac{\tau^{2} B_{1} c_{1}}{128(1+\gamma)(1+3 \gamma)}\left[B_{1}\left(4 c_{3}-4 c_{1} c_{2}+c_{1}^{3}\right)+2 B_{2} c_{1}\left(2 c_{2}-c_{1}^{2}\right)+B_{3} c_{1}^{3}\right] \\
& -\frac{\tau^{2} B_{1}^{2}}{144(1+2 \gamma)^{2}}\left[4 c_{2}^{2}+c_{1}^{4}\left(\frac{B_{2}}{B_{1}}-1\right)^{2}+4 c_{2} c_{1}^{2}\left(\frac{B_{2}}{B_{1}}-1\right)\right] \\
= & \frac{\tau^{2} B_{1}^{2}}{128(1+\gamma)(1+3 \gamma)}\left\{\left[\left(4 c_{1} c_{3}-4 c_{1}^{2} c_{2}+c_{1}^{4}\right)+\frac{2 B_{2} c_{1}^{2}}{B_{1}}\left(2 c_{2}-c_{1}^{2}\right)+\frac{B_{3}}{B_{1}} c_{1}^{4}\right]\right. \\
& \left.-\frac{8}{9} \frac{(1+\gamma)(1+3 \gamma)}{(1+2 \gamma)^{2}}\left[4 c_{2}^{2}+c_{1}^{4}\left(\frac{B_{2}}{B_{1}}-1\right)^{2}+4 c_{2} c_{1}^{2}\left(\frac{B_{2}}{B_{1}}-1\right)\right]\right\},
\end{aligned}
$$

which yields

$$
\begin{aligned}
\left|a_{2} a_{4}-a_{3}^{2}\right|= & T \mid 4 c_{1} c_{3}+c_{1}^{4}\left[1-2 \frac{B_{2}}{B_{1}}-p\left(\frac{B_{2}}{B_{1}}-1\right)^{2}+\frac{B_{3}}{B_{1}}\right]-4 p c_{2}^{2} \\
& -4 c_{1}^{2} c_{2}\left[1-\frac{B_{2}}{B_{1}}+p\left(\frac{B_{2}}{B_{1}}-1\right)\right] \mid,
\end{aligned}
$$

where

$$
T=\frac{|\tau|^{2} B_{1}^{2}}{128(1+\gamma)(1+3 \gamma)} \quad \text { and } \quad p=\frac{8}{9} \frac{(1+\gamma)(1+3 \gamma)}{(1+2 \gamma)^{2}} .
$$

It can be easily verified that $p \in\left[\frac{64}{81}, \frac{8}{9}\right]$ for $0 \leq \gamma \leq 1$.

Let

$$
\begin{aligned}
& d_{1}=4, \quad d_{2}=-4\left[1-\frac{B_{2}}{B_{1}}+p\left(\frac{B_{2}}{B_{1}}-1\right)\right], \\
& d_{3}=-4 p, \quad d_{4}=1-2 \frac{B_{2}}{B_{1}}-p\left(\frac{B_{2}}{B_{1}}-1\right)^{2}+\frac{B_{3}}{B_{1}} .
\end{aligned}
$$


Then (26) becomes

$$
\left|a_{2} a_{4}-a_{3}^{2}\right|=T\left|d_{1} c_{1} c_{3}+d_{2} c_{1}^{2} c_{2}+d_{3} c_{2}^{2}+d_{4} c_{1}^{4}\right|
$$

It follows that

$$
\begin{aligned}
\left|a_{2} a_{4}-a_{3}^{2}\right|= & \frac{T}{4} \mid c^{4}\left(d_{1}+2 d_{2}+d_{3}+4 d_{4}\right)+2 x c^{2}\left(4-c^{2}\right)\left(d_{1}+d_{2}+d_{3}\right) \\
& +\left(4-c^{2}\right) x^{2}\left(-d_{1} c^{2}+d_{3}\left(4-c^{2}\right)\right)+2 d_{1} c\left(4-c^{2}\right)\left(1-|x|^{2}\right) z \mid .
\end{aligned}
$$

Application of the triangle inequality, replacement of $|x|$ by $\mu$ and substituting the values of $d_{1}, d_{2}, d_{3}$ and $d_{4}$ from (27) yield

$$
\begin{aligned}
\left|a_{2} a_{4}-a_{3}^{2}\right| \leq & \frac{T}{4}\left[4 c^{4}\left|\frac{B_{3}}{B_{1}}-p \frac{B_{2}^{2}}{B_{1}^{2}}\right|+8\left|\frac{B_{2}}{B_{1}}\right| \mu c^{2}\left(4-c^{2}\right)(1-p)\right. \\
& \left.+\left(4-c^{2}\right) \mu^{2}\left(4 c^{2}+4 p\left(4-c^{2}\right)\right)+8 c\left(4-c^{2}\right)\left(1-\mu^{2}\right)\right] \\
= & T\left[c^{4}\left|\frac{B_{3}}{B_{1}}-p \frac{B_{2}^{2}}{B_{1}^{2}}\right|+2 c\left(4-c^{2}\right)+2 \mu\left|\frac{B_{2}}{B_{1}}\right| c^{2}\left(4-c^{2}\right)(1-p)\right. \\
& \left.+\mu^{2}\left(4-c^{2}\right)(1-p)(c-\alpha)(c-\beta)\right] \\
\equiv & F(c, \mu)
\end{aligned}
$$

where $\alpha=2, \beta=2 p /(1-p)>2$.

Similarly as in the previous proofs, it can be shown that $F(c, \mu)$ is an increasing function of $\mu$ for $0<\mu<1$. So, for fixed $c \in[0,2]$, let

$$
\max F(c, \mu)=F(c, 1) \equiv G(c),
$$

which is

$$
\begin{aligned}
G(c)= & T\left\{c^{4}\left[\left|\frac{B_{3}}{B_{1}}-p \frac{B_{2}^{2}}{B_{1}^{2}}\right|-(1-p)\left(2\left|\frac{B_{2}}{B_{1}}\right|+1\right)\right]\right. \\
& \left.+4 c^{2}\left[2\left|\frac{B_{2}}{B_{1}}\right|(1-p)+1-2 p\right]+16 p\right\} .
\end{aligned}
$$

Let

$$
\begin{aligned}
& P=\left|\frac{B_{3}}{B_{1}}-p \frac{B_{2}^{2}}{B_{1}^{2}}\right|-(1-p)\left(2\left|\frac{B_{2}}{B_{1}}\right|+1\right), \\
& Q=4\left[2\left|\frac{B_{2}}{B_{1}}\right|(1-p)+1-2 p\right], \\
& R=16 p .
\end{aligned}
$$


Using (16), we have

$$
\left|a_{2} a_{4}-a_{3}^{2}\right| \leq T \begin{cases}R, & Q \leq 0, P \leq-\frac{Q}{4} \\ 16 P+4 Q+R, & Q \geq 0, P \geq-\frac{Q}{8} \text { or } Q \leq 0, P \geq-\frac{Q}{4} \\ \frac{4 P R-Q^{2}}{4 P}, & Q>0, P \leq-\frac{Q}{8}\end{cases}
$$

where $P, Q, R$ are given in (30).

Remark 3 For the choice $\varphi(z):=(1+A z) /(1+B z)$ with $-1 \leq B<A \leq 1$, Theorem 3 reduces to [36, Theorem 2.1].

Definition 4 Let $\varphi: \mathbb{D} \rightarrow \mathbb{C}$ be analytic, and let $\varphi(z)$ be as given in (6). For a fixed real number $\alpha$, the function $f \in \mathcal{A}$ is in the class $\mathcal{G}_{\alpha}(\varphi)$ if it satisfies the following subordination:

$$
(1-\alpha) f^{\prime}(z)+\alpha\left(1+\frac{z f^{\prime \prime}(z)}{f^{\prime}(z)}\right) \prec \varphi(z)
$$

Al-Amiri and Reade [37] introduced the class $\mathcal{G}_{\alpha}:=\mathcal{G}_{\alpha}((1+z) /(1-z))$ and they showed that $\mathcal{G}_{\alpha} \subset \mathcal{S}$ for $\alpha<0$. Univalence of the functions in the class $\mathcal{G}_{\alpha}$ was also investigated in $[38,39]$. Singh et al. also obtained the bound for the second Hankel determinant of functions in $\mathcal{G}_{\alpha}$. The following theorem provides a bound for the second Hankel determinant of the functions in the class $\mathcal{G}_{\alpha}(\varphi)$.

Theorem 4 Let the function $f$ given by (1) be in the class $\mathcal{G}_{\alpha}(\varphi), 0 \leq \alpha \leq 1$. Also, let

$$
p=\frac{8}{9} \frac{(1+2 \alpha)}{(1+\alpha)}
$$

1. If $B_{1}, B_{2}$ and $B_{3}$ satisfy the conditions

$$
\begin{aligned}
& B_{1}^{2} \alpha(3-2 p)+2\left|B_{2}\right|(1+\alpha-p)+B_{1}(1+\alpha-2 p) \leq 0 \\
& \left|B_{1}^{4} \alpha(2 \alpha-1-p \alpha)+\alpha B_{1}^{2} B_{2}(3-2 p)+(\alpha+1) B_{1} B_{3}-p B_{2}^{2}\right|-p B_{1}^{2} \leq 0,
\end{aligned}
$$

then the second Hankel determinant satisfies

$$
\left|a_{2} a_{4}-a_{3}^{2}\right| \leq \frac{B_{1}^{2}}{9(1+\alpha)^{2}}
$$

2. If $B_{1}, B_{2}$ and $B_{3}$ satisfy the conditions

$$
\begin{aligned}
& B_{1}^{2} \alpha(3-2 p)+2\left|B_{2}\right|(1+\alpha-p)+B_{1}(1+\alpha-2 p) \geq 0, \\
& 2\left|B_{1}^{4} \alpha(2 \alpha-1-p \alpha)+\alpha B_{1}^{2} B_{2}(3-2 p)+(\alpha+1) B_{1} B_{3}-p B_{2}^{2}\right|-B_{1}^{3} \alpha(3-2 p) \\
& \quad-2(1+\alpha-p) B_{1}\left|B_{2}\right|-(\alpha+1) B_{1}^{2} \geq 0,
\end{aligned}
$$

or

$$
\begin{aligned}
& B_{1}^{2} \alpha(3-2 p)+2\left|B_{2}\right|(1+\alpha-p)+B_{1}(1+\alpha-2 p) \leq 0 \\
& \left|B_{1}^{4} \alpha(2 \alpha-1-p \alpha)+\alpha B_{1}^{2} B_{2}(3-2 p)+(\alpha+1) B_{1} B_{3}-p B_{2}^{2}\right|-p B_{1}^{2} \geq 0
\end{aligned}
$$


then the second Hankel determinant satisfies

$$
\left|a_{2} a_{4}-a_{3}^{2}\right| \leq \frac{\left|B_{1}^{4} \alpha(2 \alpha-1-p \alpha)+\alpha B_{1}^{2} B_{2}(3-2 p)+(\alpha+1) B_{1} B_{3}-p B_{2}^{2}\right|}{8(1+\alpha)(1+2 \alpha)} .
$$

3. If $B_{1}, B_{2}$ and $B_{3}$ satisfy the conditions

$$
\begin{aligned}
& B_{1}^{2} \alpha(3-2 p)+2\left|B_{2}\right|(1+\alpha-p)+B_{1}(1+\alpha-2 p)>0, \\
& 2\left|B_{1}^{4} \alpha(2 \alpha-1-p \alpha)+\alpha B_{1}^{2} B_{2}(3-2 p)+(\alpha+1) B_{1} B_{3}-p B_{2}^{2}\right|-B_{1}^{3} \alpha(3-2 p) \\
& \quad-2(1+\alpha-p) B_{1}\left|B_{2}\right|-(\alpha+1) B_{1}^{2} \leq 0,
\end{aligned}
$$

then the second Hankel determinant satisfies

$$
\begin{aligned}
\mid a_{2} a_{4} & -a_{3}^{2} \mid \\
\leq & \frac{B_{1}^{2}}{32(1+\alpha)(1+2 \alpha)} \\
& \times\left[\begin{array}{c}
4 p-\frac{\left[B_{1}^{2} \alpha(3-2 p)+2\left|B_{2}\right|(1+\alpha-p)+B_{1}(1+\alpha-2 p)\right]^{2}}{\left|B_{1}^{4} \alpha(2 \alpha-1-p \alpha)+\alpha B_{1}^{2} B_{2}(3-2 p)+(\alpha+1) B_{1} B_{3}-p B_{2}^{2}\right|} \\
-B_{1}^{3} \alpha(3-2 p)-(1+\alpha-p) B_{1}\left(2\left|B_{2}\right|+B_{1}\right)
\end{array}\right] .
\end{aligned}
$$

Proof For $f \in \mathcal{G}_{\alpha}(\varphi)$, a calculation shows that

$$
\begin{aligned}
& \left|a_{2} a_{4}-a_{3}^{2}\right| \\
& =T \mid 4(1+\alpha) B_{1} c_{1} c_{3}+c_{1}^{4}\left[-3 \alpha B_{1}^{2}+\alpha(2 \alpha-1) B_{1}^{3}+B_{1}(1+\alpha)+3 \alpha B_{1} B_{2}\right. \\
& \left.\quad+(1+\alpha)\left(B_{3}-2 B_{2}\right)-p \frac{\left(\alpha B_{1}^{2}-B_{1}+B_{2}\right)^{2}}{B_{1}}\right]-4 p B_{1} c_{2}^{2} \\
& \quad+2 c_{1}^{2} c_{2}\left[-2(1+\alpha) B_{1}+3 \alpha B_{1}^{2}+2(1+\alpha) B_{2}-2 p\left(\alpha B_{1}^{2}-B_{1}+B_{2}\right)\right] \mid
\end{aligned}
$$

where

$$
T=\frac{B_{1}}{128(1+\alpha)(1+2 \alpha)} \quad \text { and } \quad p=\frac{8}{9} \frac{(1+2 \alpha)}{(1+\alpha)} .
$$

It can be easily verified that for $0 \leq \alpha \leq 1, p \in\left[\frac{8}{9}, \frac{4}{3}\right]$. Let

$$
\begin{aligned}
d_{1}= & 4(1+\alpha) B_{1} \\
d_{2}= & 2\left[-2(1+\alpha) B_{1}+3 \alpha B_{1}^{2}+2(1+\alpha) B_{2}-2 p\left(\alpha B_{1}^{2}-B_{1}+B_{2}\right)\right], \\
d_{3}= & -4 p B_{1} \\
d_{4}= & -3 \alpha B_{1}^{2}+\alpha(2 \alpha-1) B_{1}^{3}+B_{1}(1+\alpha)+3 \alpha B_{1} B_{2} \\
& +(1+\alpha)\left(B_{3}-2 B_{2}\right)-p \frac{\left(\alpha B_{1}^{2}-B_{1}+B_{2}\right)^{2}}{B_{1}} .
\end{aligned}
$$


Then

$$
\left|a_{2} a_{4}-a_{3}^{2}\right|=T\left|d_{1} c_{1} c_{3}+d_{2} c_{1}^{2} c_{2}+d_{3} c_{2}^{2}+d_{4} c_{1}^{4}\right| .
$$

Similarly as in earlier theorems, it follows that

$$
\begin{aligned}
\left|a_{2} a_{4}-a_{3}^{2}\right|= & \frac{T}{4} \mid c^{4}\left(d_{1}+2 d_{2}+d_{3}+4 d_{4}\right)+2 x c^{2}\left(4-c^{2}\right)\left(d_{1}+d_{2}+d_{3}\right) \\
& +\left(4-c^{2}\right) x^{2}\left(-d_{1} c^{2}+d_{3}\left(4-c^{2}\right)\right)+2 d_{1} c\left(4-c^{2}\right)\left(1-|x|^{2}\right) z \mid \\
\leq & T\left[c^{4} \mid B_{1}^{3} \alpha(2 \alpha-1-p \alpha)+\alpha B_{1} B_{2}(3-2 p)\right. \\
& +(\alpha+1) B_{3}-p \frac{B_{2}^{2}}{B_{1}} \mid+\mu c^{2}\left(4-c^{2}\right)\left[B_{1}^{2} \alpha(3-2 p)\right. \\
& \left.+2\left|B_{2}\right|(1+\alpha-p)\right]+2 c\left(4-c^{2}\right) B_{1}(1+\alpha) \\
& \left.+\mu^{2}\left(4-c^{2}\right) B_{1}(1+\alpha-p)(c-2)\left(c-\frac{2 p}{1+\alpha-p}\right)\right] \\
\equiv & F(c, \mu)
\end{aligned}
$$

and for fixed $c \in[0,2], \max F(c, \mu)=F(c, 1) \equiv G(c)$ with

$$
\begin{aligned}
G(c)= & T\left[c ^ { 4 } \left[\left|B_{1}^{3} \alpha(2 \alpha-1-p \alpha)+\alpha B_{1} B_{2}(3-2 p)+(\alpha+1) B_{3}-p \frac{B_{2}^{2}}{B_{1}}\right|\right.\right. \\
& \left.-B_{1}^{2} \alpha(3-2 p)-(1+\alpha-p)\left(2\left|B_{2}\right|+B_{1}\right)\right]+4 c^{2}\left[B_{1}^{2} \alpha(3-2 p)\right. \\
& \left.\left.+2\left|B_{2}\right|(1+\alpha-p)+B_{1}(1+\alpha-2 p)\right]+16 p B_{1}\right] .
\end{aligned}
$$

Let

$$
\begin{aligned}
P= & \left|B_{1}^{3} \alpha(2 \alpha-1-p \alpha)+\alpha B_{1} B_{2}(3-2 p)+(\alpha+1) B_{3}-p \frac{B_{2}^{2}}{B_{1}}\right| \\
& -B_{1}^{2} \alpha(3-2 p)-(1+\alpha-p)\left(2\left|B_{2}\right|+B_{1}\right), \\
Q= & 4\left[B_{1}^{2} \alpha(3-2 p)+2\left|B_{2}\right|(1+\alpha-p)+B_{1}(1+\alpha-2 p)\right], \\
R= & 16 p B_{1} .
\end{aligned}
$$

By using (16), we have

$$
\left|a_{2} a_{4}-a_{3}^{2}\right| \leq T \begin{cases}R, & Q \leq 0, P \leq-\frac{Q}{4} \\ 16 P+4 Q+R, & Q \geq 0, P \geq-\frac{Q}{8} \text { or } Q \leq 0, P \geq-\frac{Q}{4} \\ \frac{4 P R-Q^{2}}{4 P}, & Q>0, P \leq-\frac{Q}{8}\end{cases}
$$

where $P, Q, R$ are given in (35). 
Remark 4 For $\alpha=1$, Theorem 4 reduces to Theorem 2 . For $0 \leq \alpha<1$, let $\varphi(z):=(1+(1-$ $2 \alpha) z) /(1-z)$. For this function $\varphi, B_{1}=B_{2}=B_{3}=2(1-\alpha)$. In this case, Theorem 4 reduces to [40, Theorem 3.1].

\section{Competing interests}

The authors declare that they have no competing interests.

Authors' contributions

All authors jointly worked on the results and they read and approved the final manuscript.

\section{Author details}

${ }^{1}$ School of Mathematical Sciences, Universiti Sains Malaysia, 11800 USM, Penang, Malaysia. ${ }^{2}$ Department of Mathematics, University of Delhi, Delhi, 110007, India.

\section{Acknowledgements}

The work presented here was supported in part by research grants from Universiti Sains Malaysia (FRGS grants) and University of Delhi as well as MyBrain MyPhD programme of the Ministry of Higher Education, Malaysia.

Received: 11 December 2012 Accepted: 11 March 2013 Published: 5 June 2013

\section{References}

1. Cantor, DG: Power series with integral coefficients. Bull. Am. Math. Soc. 69, 362-366 (1963)

2. Wilson, R: Determinantal criteria for meromorphic functions. Proc. Lond. Math. Soc. 4, 357-374 (1954)

3. Vein, R, Dale, P: Determinants and Their Applications in Mathematical Physics. Applied Mathematical Sciences, vol. 134. Springer, New York (1999)

4. Pommerenke, C: On the coefficients and Hankel determinants of univalent functions. J. Lond. Math. Soc. 41, 111-122 (1966)

5. Pommerenke, C: On the Hankel determinants of univalent functions. Mathematika 14, 108-112 (1967)

6. Hayman, WK: On the second Hankel determinant of mean univalent functions. Proc. Lond. Math. Soc. 18, 77-94 (1968)

7. Noonan, JW, Thomas, DK: On the Hankel determinants of areally mean $p$-valent functions. Proc. Lond. Math. Soc. 25 , 503-524 (1972)

8. Noonan, JW: Coefficient differences and Hankel determinants of areally mean $p$-valent functions. Proc. Am. Math. Soc. 46, 29-37 (1974)

9. Noonan, JW, Thomas, DK: On the second Hankel determinant of areally mean $p$-valent functions. Trans. Am. Math Soc. 223, 337-346 (1976)

10. Elhosh, MM: On the second Hankel determinant of univalent functions. Bull. Malays. Math. Soc. 9(1), $23-25$ (1986)

11. Elhosh, MM: On the second Hankel determinant of close-to-convex functions. Bull. Malays. Math. Soc. 9(2), 67-68 (1986)

12. Noor, Kl: Higher order close-to-convex functions. Math. Jpn. 37(1), 1-8 (1992)

13. Noor, Kl: On the Hankel determinant problem for strongly close-to-convex functions. J. Nat. Geom. 11(1), 29-34 (1997)

14. Noor, Kl: On certain analytic functions related with strongly close-to-convex functions. Appl. Math. Comput. 197(1), 149-157 (2008)

15. Noor, Kl, Al-Bany, SA: On Bazilevic functions. Int. J. Math. Math. Sci. 10(1), 79-88 (1987)

16. Noor, Kl: On analytic functions related with functions of bounded boundary rotation. Comment. Math. Univ. St. Pauli 30(2), 113-118 (1981)

17. Noor, Kl: On meromorphic functions of bounded boundary rotation. Caribb. J. Math. 1(3), 95-103 (1982)

18. Noor, Kl: Hankel determinant problem for the class of functions with bounded boundary rotation. Rev. Roum. Math. Pures Appl. 28(8), 731-739 (1983)

19. Noor, Kl, Al-Naggar, IMA: On the Hankel determinant problem. J. Nat. Geom. 14(2), 133-140 (1998)

20. Arif, M, Noor, Kl, Raza, M: Hankel determinant problem of a subclass of analytic functions. J. Inequal. Appl. 2012, Article ID 22 (2012)

21. Hayami, T, Owa, S: Generalized Hankel determinant for certain classes. Int. J. Math. Anal. 4(49-52), $2573-2585$ (2010)

22. Hayami, T, Owa, S: Applications of Hankel determinant for $p$-valently starlike and convex functions of order $\alpha$. Far East J. Appl. Math. 46(1), 1-23 (2010)

23. Hayami, T, Owa, S: Hankel determinant for $p$-valently starlike and convex functions of order $\alpha$. Gen. Math. 17(4), 29-44 (2009)

24. Janteng, A, Halim, SA, Darus, M: Hankel determinant for starlike and convex functions. Int. J. Math. Anal. 1(13-16), 619-625 (2007)

25. Mishra, AK, Gochhayat, P: Second Hankel determinant for a class of analytic functions defined by fractional derivative. Int. J. Math. Math. Sci. 2008, Article ID 153280 (2008)

26. Mohamed, N, Mohamad, D, Cik Soh, S: Second Hankel determinant for certain generalized classes of analytic functions. Int. J. Math. Anal. 6(17-20), 807-812 (2012)

27. Murugusundaramoorthy, G, Magesh, N: Coefficient inequalities for certain classes of analytic functions associated with Hankel determinant. Bull. Math. Anal. Appl. 1(3), 85-89 (2009)

28. Ali, RM, Lee, SK, Ravichandran, V, Supramaniam, S: The Fekete-Szegö coefficient functional for transforms of analytic functions. Bull. Iran. Math. Soc. 35(2), 119-142, 276 (2009) 
29. Ali, RM, Ravichandran, V, Seenivasagan, N: Coefficient bounds for $p$-valent functions. Appl. Math. Comput. 187(1), 35-46 (2007)

30. Ma, WC, Minda, D: A unified treatment of some special classes of univalent functions. In: Proceedings of the Conference on Complex Analysis, Tianjin, 1992. Conf. Proc. Lecture Notes Anal., vol. I, pp. 157-169. International Press, Cambridge (1922)

31. Duren, PL: Univalent Functions. Grundlehren der Mathematischen Wissenschaften, vol. 259. Springer, New York (1983)

32. Grenander, U, Szegö, G: Toeplitz Forms and Their Applications. California Monographs in Mathematical Sciences. University of California Press, Berkeley (1958)

33. Rønning, F: Uniformly convex functions and a corresponding class of starlike functions. Proc. Am. Math. Soc. 118(1), 189-196 (1993)

34. Ali, RM, Ravichandran, V: Uniformly convex and uniformly starlike functions. Math. News Lett. 21(1), 16-30 (2011)

35. Sokół J, Stankiewicz, J: Radius of convexity of some subclasses of strongly starlike functions. Zeszyty Nauk. Politech. Rzeszowskiej Mat. 19, 101-105 (1996)

36. Bansal, D: Upper bound of second Hankel determinant for a new class of analytic functions. Appl. Math. Lett. 26(1), 103-107 (2013)

37. Al-Amiri, HS, Reade, $\mathrm{MO}$ : On a linear combination of some expressions in the theory of the univalent functions. Monatshefte Math. 80(4), 257-264 (1975)

38. Singh, S, Gupta, S, Singh, S: On a problem of univalence of functions satisfying a differential inequality. Math. Inequal. Appl. 10(1), 95-98 (2007)

39. Singh, V, Singh, S, Gupta, S: A problem in the theory of univalent functions. Integral Transforms Spec. Funct. 16(2), 179-186 (2005)

40. Verma, S, Gupta, S, Singh, S: Bounds of Hankel determinant for a class of univalent functions. Int. J. Math. Math. Sci. 2012, Article ID 147842 (2012)

doi:10.1186/1029-242X-2013-281

Cite this article as: Lee et al.: Bounds for the second Hankel determinant of certain univalent functions. Journal of Inequalities and Applications 2013 2013:281.

\section{Submit your manuscript to a SpringerOpen ${ }^{\odot}$ journal and benefit from:}

- Convenient online submission

- Rigorous peer review

- Immediate publication on acceptance

- Open access: articles freely available online

- High visibility within the field

- Retaining the copyright to your article 